\title{
Axiomatic summary and deductions from Hering's principles of visual direction
}

\author{
HIROSHI ONO \\ York University, Toronto, Ontario, Canada
}

\begin{abstract}
Hering's principles of visual direction are summarized axiomatically, and deductions are presented. The deductions allow one to predict when veridical or nonveridical judgments of visual direction should occur and when apparent movement should be seen. These predictions agree with the results obtained in special viewing conditions. However, one of the limitations of the principles is that some of the predictions fail in "normal" viewing conditions.
\end{abstract}

If we had only one eye as do the Cyclopes, sensing the directions of objects would be a simple matter. If the single eye could not rotate in its socket, there would be a one-to-one correspondence between the directions of objects in space relative to the eye (and head) and the retinal locations of the images. Thus, image locations on the retina relative to the fovea would provide complete information about the directions of objects in space relative to the head (egocentric direction). If the single eye could rotate, the retinal image locations together with information about the angular position of the eye would provide sufficient information for egocentric direction.

Hering $(1879 / 1942)$ proposed that we behave as if we have a single "cyclopean" eye from which we judge visual direction. According to Hering, the direction of an object from the cyclopean eye is specified by the angular position of the two eyes and the loci of the images on the two retinae. The way in which these two factors determine visual direction from the cyclopean eye has been restated several times (e.g., Fry, 1950; Howard \& Templeton, 1966; Linksz, 1952; Ogle, 1962), but a simpler and complete restatement can be made in terms of the following axiomatic principles of visual direction. These principles are:

(1) Visual directions of objects are judged as if from the cyclopean eye located midway between the two eyes. ${ }^{1}$

(2) An object which stimulates the center of a fovea in either eye or the centers of the fovea in both eyes will be seen on a line passing through the cyclopean eye and the point of intersection of the two lines of sight (visual axes).

(3) An object which stimulates any other position on either retina will be seen from the cyclopean eye with a particular angular deviation from the line

This research was supported by Grant $\mathrm{A0296}$ from the National Research Council of Canada. The author wishes to thank his many colleagues in perception at York University for helpful comments on an earlier version of this paper. passing through the cyclopean eye and the intersection of the two lines of sight-the deviation being equal to the angle subtended by the line of sight and the line from the object to the eye (visual line).

Hering's "law" which deals with stimulation of corresponding retinal points and identical visual directions is not included among the preceding principles (cf. Howard \& Templeton, 1966; Ogle, 1962; Ono, 1975), since it can be derived from Principles 2 and 3 as follows. An object (or two different objects) which stimulates the centers of the two foveae will be seen in identical visual directions because stimulation of either fovea should lead to a visual direction on the line passing through the cyclopean eye and the point of intersection of the two lines of sight (Principle 2). When the relative angular directions of an object (or two different objects) with respect to the lines of sight of the two eyes are the same, the object stimulates corresponding points on the two retinae. According to Principle 3, stimulation of the two eyes in the preceding manner should lead to the same visual direction, because any object with the same relative angular deviations from the two lines of sight should have the same visual direction. Thus, according to Principles 2 and 3, stimulation of corresponding retinal points leads to identical visual directions.

Two other deductions from the principles are illustrated in Figures 1 and 2. According to the principles, the three situations illustrated in Figure 1 give rise to veridical visual directions of objects. But for other situations, the principles predict illusory or nonveridical visual directions, e.g., Hering's classical demonstration with a marker on a window pane aligned to a tree top with respect to one eye and a chimney with respect to the other eye. Figure 2 illustrates another situation where visual directions are nonveridical.

Figures $1 \mathrm{a}$ and $1 \mathrm{~b}$ illustrate how visual direction is determined when an observer fixates on an object. Since the centers of the foveae of both retinae are being stimulated, the object will be seen on the line passing through the intersection of the two lines of 


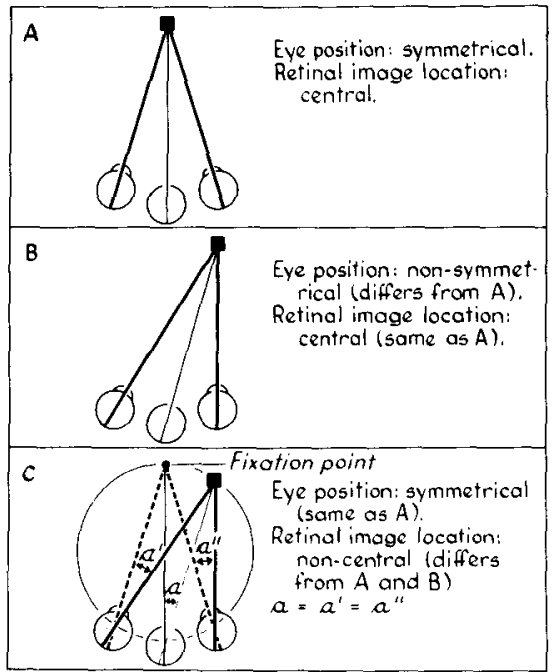

Figure 1. Three stimulus situations in which veridical visual direction is predicted by the principles.

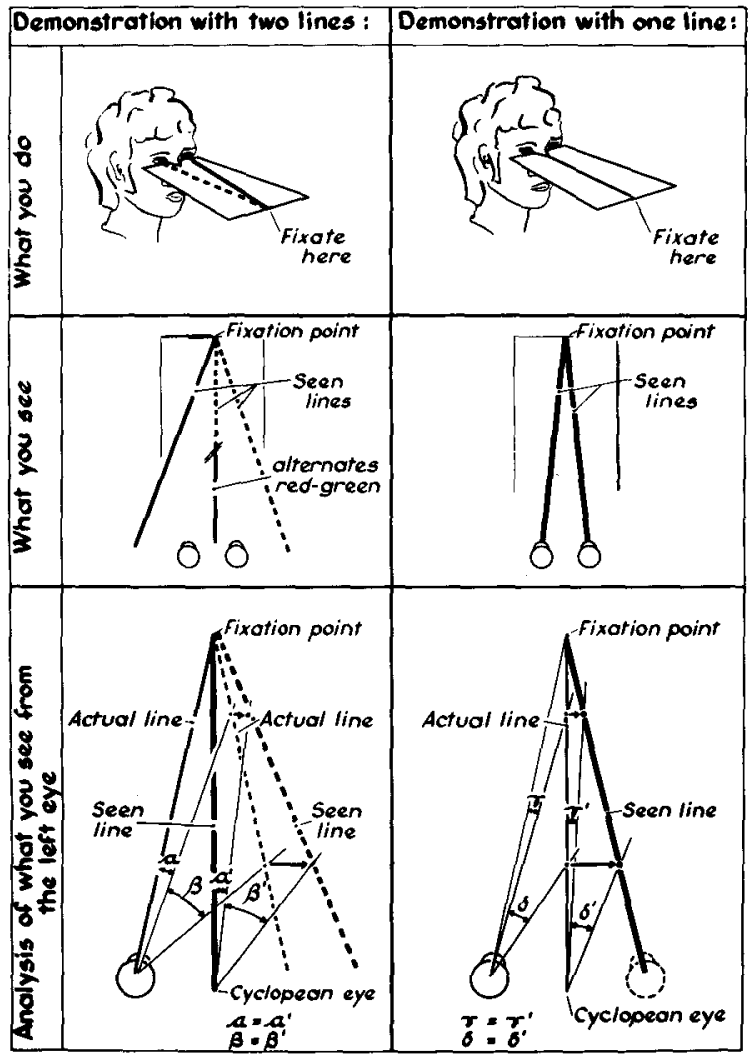

Figure 2. The stimulus which produces nonveridical visual directions and an analysis in terms of the principles of visual direction.

sight and the cyclopean eye (Principles 1 and 2). (The difference in visual directions in Figures $1 \mathrm{a}$ and $1 \mathrm{~b}$ makes clear that the location of images on the two retinae is insufficient information for determining visual direction.) In Figure $1 c$, the object is not stimulating the centers of the foveae but subtends the same angle from the two lines of sight. The object will be seen from the cyclopean eye in the direction which matches the angle of entry of each eye (Principle 3).

The demonstration card illustrated in Figure 2 provides some illusory phenomena predicted by the principles. The card is similar to the stimuli used by Le Conte $(1871,1881)$, but modified, using two colored lines, red and green. In Figure 2, a red line is represented by a solid line, and a green line by a dotted line. The use of differently colored lines clarifies what happens perceptually to the different inputs to the two eyes, which will be explained shortly. The card should be held horizontally just below the eyes, as shown on the left side of Figure 2, and the apex of the triangle should be fixated so that each line is pointing directly to one eye. Although there are individual differences in interocular distances, the card can be made so that most people can use it. People with a small interocular distance should hold the card close to the face, and people with a larger interocular distance should hold it slightly away from the face.

According to Principle 2, one should see a line pointing to the base of the nose, or between the eyes. For most people, the line will alternate in color-red or green. The alternation shows that the line that points to the base of the nose is the outcome of stimulation of either eye. (Using a solid line and dotted line as in Figure 2 is not as effective; our informal experiment indicated that such stimuli do not rival as frequently as the two differently colored lines.) The fixated apex should appear straight ahead, because convergence is symmetrical and image location is central. Because each colored line is stimulating the retina near the center of the fovea, the line should also appear to be straight ahead of the cyclopean eye.

Notice that the other two lines one sees do not appear to be pointing directly to the eyes. As a matter of fact, none of the three lines one sees appears to be pointing directly to the eyes, despite the fact that two lines are actually pointing to the eyes. One line appears to be pointing to the nose and the other two lines appear to be pointing to the outside of each eye. The explanation of the apparent positions of the two outside lines can be derived from the principles. The bottom left of Figure 2 considers stimulation to the left eye. The red line is pointing to the center of the fovea and should appear to be pointing to the cyclopean eye (Principle 2). The figure shows how the red line is displaced visually. Apparent rotation of the green line (pivoting around the apex) occurs because near segments (or points) of the green line have a greater angular separation from the left eye's line of sight (the actual red line) than do far segments (or points), i.e., $\beta>\alpha$ in Figure 2. According to Principle 3 , this angular separation is maintained at the cyclopean eye, i.e., $\beta^{\prime}>\alpha^{\prime}$. If the distance of the segments is more or less correctly perceived, the 
green line will be displaced in the same direction as the visual displacement of the red line. The figure shows where the green line should appear, to the outside and right of the right eye. If we go through the same reasoning with respect to stimulation of the right eye, we can determine where the red line should appear, i.e., to the outside and left of the left eye.

Thus, when each eye has a line pointing to it, one does not perceive it that way. We can also produce a stimulus that appears to point to the two eyes, although it actually does not. A demonstration card can be made to illustrate this (see the top right of Figure 2). If the card is held so that the black line is pointing to the base of the nose and the far end of the line is fixated, you should see two lines, one pointing to each eye. The explanation of the apparent positions of these two lines is the same as the explanation for the two lines that did not appear to point to the eyes (Principle 3 ).

In agreement with the predictions from the principles, the demonstration indicated that all points in front of the fixation point appeared in illusory locations. If the lines in the demonstration were to be extended beyond the fixation point, it can also be shown that all the points behind a fixation point would be mislocalized. ${ }^{2}$ Furthermore, the principles predict that there should be an illusory movement in certain stimulus conditions where the extent of mislocalization changes with a change in eye position. Two such conditions are illustrated in Figures 3 and 4. Figure 3 illustrates how a change in accommodative vergence should produce apparent movement, and Figure 4 illustrates how alternating occlusion of the two eyes should produce apparent movement.

Figure 3 depicts a situation in which two stimuli are aligned with the viewing eye while the other eye is occluded. The far stimulus is placed slightly above

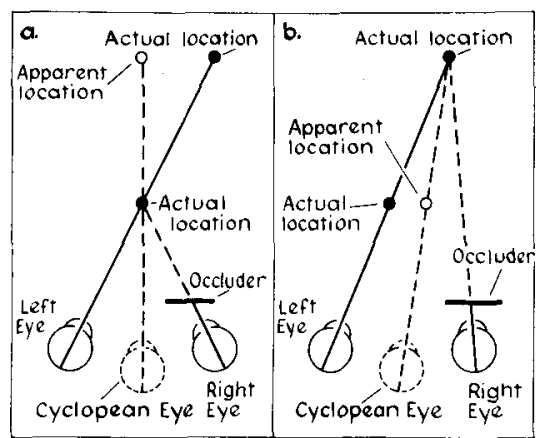

Figure 3. Apparent locations of a stimulus predicted by the principles of visual direction in an accommodative vergence situation. In (a), an observer accommodates to the near stimulus, and in (b), to the far stimulus. The difference in the apparent locations in (a) and (b) is the predicted extent of apparent movement when there is no phoria.

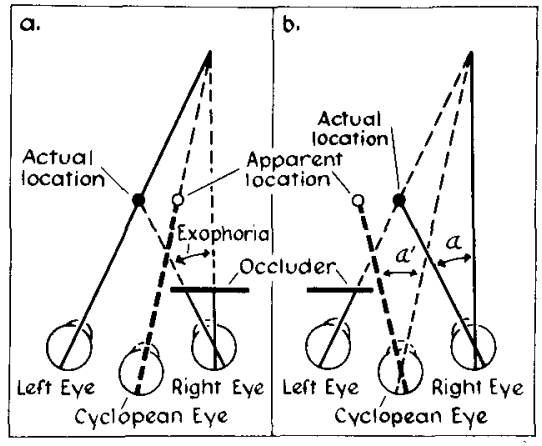

Figure 4. Apparent locations of a stimulus as a result of exophoria of the right eye. The difference in the apparent locations In (a) and (b) is the predicted extent of apparent movement.

the near one so it can be seen. In the figure, the cyclopean eye is assumed to be centrally located midway between the two eyes; the observer correctly perceives the distance of the sitmuli; and the line of sight of the viewing eye and the extension of the obstructed line of sight of the nonviewing eye intersect at one or the other stimulus. Figure $3 a$ shows the observer accommodating to the near stimulus. The near and the far stimuli should be seen straight ahead, because the imaginary line passing through the cyclopean eye and the intersection of the two lines of sight are straight ahead (Principle 2). Figure 3b shows the observer accommodating to the far stimulus. The lines of sight intersect at the far stimulus; therefore, one should see the far stimulus where it is, but the near stimulus should be displaced to the right, in accordance with Principle 2. The predicted extent of apparent movement is the difference in the apparent location before and after the eye movement, i.e., the difference in the apparent locations shown in Figures 3a and 3b.

Figure 4 depicts a situation with one stimulus in which each of the two eyes is alternately occluded. In Figure $4 a$, the right eye is occluded, and we will suppose that exophoria occurs (an outward deviation of the eye). Because the center of the fovea in the left eye is being stimulated, the predicted apparent location of the stimulus is to the right of the actual location, i.e., on an imaginary line passing through the cyclopean eye and the intersection of the two lines of sight (Principle 2). Figure $4 \mathrm{~b}$ shows the occluder in front of the left eye. When occlusion shifts, the stimulus does not stimulate the fovea of the right eye but rather a peripheral retinal location specified by a certain angle ( $\alpha$ in Figure 4) from the line of sight. According to Principle 3, this angle is projected to the cyclopean eye, thus determining the predicted apparent location of the stimulus to be on the left of the actual location. The extent of the predicted apparent movement is the difference in the predicted apparent locations shown in Figures $4 \mathrm{a}$ and $4 \mathrm{~b}$. 
Results consistent with the predictions concerning the extent of illusory movement have been found. In an accommodative vergence situation, the extent of apparent movement shown in Figure 3 is seldom found; it is generally much smaller and sometimes reported not to occur (Walls, 1951). The extent of apparent movement is smaller, because in this situation phoria occurs and the intersection of the two lines of sight is at a different location from those shown in Figures 3a and 3b. Ono, Wilkinson, Muter, and Mitson (1972) measured phoria for different subjects and used the measured amount to predict the extent of apparent movement. They found the correlation between the predicted and observed values to be .72 . When an occluder was alternated between the two eyes, as in Figure 4, the extent of apparent movement should also be a function of the amount of phoria. Ono and Gonda (1978) found the correlation between the degree of phoria and the extent of apparent movement to be .95. The high correlations justified our treating the apparent movement shown in Figures 3 and 4 as being equal to a change in egocentric direction, and provided strong confirmation for the predictions based on the principles of visual direction. A counterintuitive aspect of the predictions or the results is that the direction and magnitude of apparent movement are a function of the position of the nonviewing eye. The results, however, clearly indicated that the position of the occluded eye as well as that of the viewing eye determined the visual direction as predicted by the principles.

Diplopia is a phenomenon also predicted by the principles of visual direction and is an instance of a stimulus appearing in illusory locations. According to Principles 2 and 3 , a point in space which stimulates noncorresponding points should appear in two illusory locations. Because there are many points in space which stimulate noncorresponding points on the two retinae, double vision should be the rule, rather than the exception, according to the principles. Furthermore, because the extent of disparity varies with the change in convergence, these double images should appear to move every time convergence is changed, just as in an accommodative vergence situation. Points in space which project to corresponding points are confined only to the horopter (the horizontal section of which is a circle that passes through the nodal points of the two eyes and the fixation point). Yet we usually see only one visual world. Even if Panum's area (a region in which single vision occurs by means of sensory fusion or suppression when noncorresponding points are stimulated) is as large as $45^{\prime}$ or $1^{\circ}$ (Ono, Angus, \& Gregor, 1977), double vision should still be very frequent. ${ }^{3}$
The predictions concerning double vision or those shown in Figures 3 and 4 are readily confirmed in the laboratory, but are not easily confirmed by a naive observer under normal viewing conditions. (Perhaps, because of this, these phenomena are not well known among nonspecialists, as compared, say, to the moon illusion or induced motion.) It can be argued that not experiencing these phenomena is functional, since experiencing nonveridical locations can only interfere with daily life. One can speculate that the mechanism which processes visual direction evolved to process the location of fixated objects and that one of the functions of eye movements is to change fixation so that the correct visual direction of another object can be determined. These statements imply that the illusory visual directions of a nonfixated stimulus are epiphenomena associated with the mechanism which processes the visual direction of fixated objects. Consistent with this idea is the finding that the oculomotor system does not use illusory information in programming saccadic eye movements (Hallett \& Lightstone, 1976; Ono \& Nakamizo, 1977), i.e., saccadic eye movements to a stimulus that is mislocalized (indicated by a pointing response) are directed appropriately to the actual location rather than to the illusory location.

The fact that we do not experience some of the phenomena discussed in this paper under normal viewing conditions is not necessarily a failure of the principles, but suggests that one must postulate additional mechanisms to account for our typically not experiencing these phenomena. One can speculate that, in addition to the mechanism which processes visual direction, another mechanism has evolved to prevent experiencing these phenomena. Such a mechanism would operate under normal viewing conditions but not in the special stimulus situations discussed previously. The question remains as to what this mechanism is, and what difference in the two situations controls it.

\section{REFERENCES}

BAILEY, N. J. Locating the center of visual direction by a binocular diplopia method. American Journal of Optometry, 1958, 35, 484-495.

Barbeito, R., \& ONo, H. Four methods of locating the egocenter: A comparison of the predictive validities and reliabilities. Behavior Research Methods \& Instrumentation, 1979, 11, 31-36.

FRY, G. A. Visual perception of space. American Journal of Optometry, 1950, 27, 531-553.

Funaishi, S. Weiterers über das Zentrum der Sehrichtungen. Albrecht von Graefes Archiv für Ophthalmologie, 1926, 117, 296-303.

Hallett, P. E., \& Lightstone, A. D. Saccadic eye movements towards stimuli triggered by prior saccades. Vision Research, 1976, 16, 99-107. 
Hering, E. [Spatial sense and movements of the eye] (C. A. Radde, trans.). Baltimore: American Academy of Optometry, 1942. (Originally published, 1879).

Howard, I. P., \& TEM PLETON, W. B. Human spatial orientation. New York: Wiley, 1966.

Le Conte, J. On some phenomena of binocular vision. American Journal of Science, 1871, 1, 33-44.

Le Conte, J. Sight: An exposition of the principles of monocular and binocular vision. New York: Appleton, 1881.

Linksz. A. Physiology of the eye (Vol. 2) Vision. New York: Grune \& Stratton, 1952.

Mitchell, E. E. A review of the concept of "Panum's fusional areas." American Journal of Optometry, 1966, 43, 387.401.

Mitson, G. L., Ono, H., \& Barbeito, R. Three methods of measuring the location of the egocentre: Their reliability, comparative locations and intercorrelations. Canadian Joumal of Psychology, 1976, 30. 1-8.

Ogle, K. N. Spatial localization according to direction. In $\mathrm{H}$. Davson (Ed.), The eye (Vol. 4). London: Academic Press, 1962.

ONo, H. Directions of objects seen from the cyclopean eye. Saensu (Japanese edition of Scientific American), 1975, 5, 88-99. (The English translation appeared as Department of Psychology Reports, York University, Toronto, 1976, No. 29).

ONo, H., \& ANGus, R. G. Adaptation to sensory-motor conflict produced by the visual direction of the hand specified from the cyclopean eye. Journal of Experimental Psychology, 1974, 103. 1 -9.

Ono, H., ANgus, R., \& Gregor, P. Binocular single vision achieved by fusion and suppression. Perception \& Psychophysics, $1977,21,513-521$.

Ono, H., \& Gonda, G. Apparent movement, eye movements and phoria when two eyes alternate in viewing a stimulus. Perception, $1978,7,75-83$.
ONo, H., \& NaKamizo, S. Saccadic eye movements during changes in fixation to stimuli at different distances. Vision Research, $1977,17,233-238$.

Ono, H., Wilkinson, A., Muter, P., \& Mitson, L. Apparent movement and change in perceived location of a stimulus produced by a change in accommodative vergence. Perception \& Psychophysics, 1972, 12, 187-192.

Roelofs, C. O. Considerations on the visual egocentre. Acta Psychologica, 1959, 16, 226-234.

Walls. G. L. A theory of ocular dominance. Archives of Ophthalmalogy, 1951, 45, 387-412.

\section{NOTES}

1. Some investigators (e.g., Bailey, 1958; Funaishi, 1926; Mitson, Ono, \& Barbeito, 1976; Roelofs, 1959; Barbeito \& Ono, 1979) suggest that for some people the actual location departs from the assumed location. This conceptual eye is sometimes called: projection center, egocenter, binoculus, and visual direction center.

2. For any given fixation, the mislocalization is similar to prismatic displacement to which sensory-motor adaptation can occur (Ono \& Angus, 1974).

3. The extent of Panum's area usually reported is considerably smaller-sometimes as small as $\mathbf{2}$ min. For a summary of the results from different studies, see Mitchell (1966).

(Received for publication June 1, 1978; revision accepted December 22, 1978.) 